\title{
The supraduodenal pedicle can be a useful landmark of the common bile duct in laparoscopic cholecystectomy
}

\author{
Lei Wang, Hui Hou \\ Department of General Surgery, The Second Affiliated Hospital of Anhui Medical University, Hefei, China
}

Videosurgery Miniinv 2021; 16 (3): 620-621

DOI: https://doi.org/10.5114/wiitm.2021.108213

\begin{abstract}
This investigation was undertaken to determine whether the supraduodenal pedicle (SP) can assist in defining the location of the common bile duct (CBD) during laparoscopic cholecystectomy $(L C)$. The supraduodenal pedicle was recognizable in 100/122 (82\%) consecutive patients undergoing LC in this hospital. The SP displayed either thin (9/100,9\%) or obvious thick vessels (91/100, 91\%), 38/100 (38\%) cases showed a single branch vessel and 62/100 (62\%) cases showed two or more branches. The CBD and SP were both present in 68/100 (68\%) patients. The right most branch of the SP ran along the ventral $(40 / 68,58.8 \%)$ or the left edge $(28 / 68,41.2 \%)$ of the CBD. In 32 cases with an invisible $C B D$ but a visible $S P$, the $C B D$ was successfully found close to the right most branch of the SP in all patients after slightly separating them. The SP can be a useful landmark to quickly and accurately locate the CBD in $L C$.
\end{abstract}

Key words: supraduodenal pedicle, anatomic landmark, laparoscopic cholecystectomy, bile duct injury.

Accidental bile duct injury is a serious complication that cannot be completely avoided in laparoscopic cholecystectomy (LC). Its incidence is relatively high, especially for patients with severe tissue edema and dense adhesion caused by inflammation [1, 2]. A common error is mistaking the common bile duct (CBD) for low confluence of the cystic duct. Quick and accurate CBD location is the key to avoid CBD injury.

As the CBD constantly passes through the first part of the duodenum, it is possible to find the CBD on the upper edge of the first part of the duodenum where it is far away from the severe edema and adhesion in Calot's triangle [3]. Some constant vessels in this region were observed, which aided in recognition of the CBD during laparoscopy. These vessels were below the serosa layer of the hepatoduodenal ligament and have seldom been described in the literature and classic anatomy textbooks. Their course was different from the prepyloric vein.
In 1911, Wilkie [4] for the first time described a distinctive artery below the serosa layer of the hepatoduodenal ligament above the first part of the duodenum which he called the supraduodenal artery. Hentati further introduced the concept of the "supraduodenal pedicle (SP)" through cadaveric and radiologic investigation, and found that the blood current of the SP originated from the right gastric artery or proper hepatic artery. In order to take the transitional character of the duodenal bulb into account, Hentati divided the supraduodenal pedicle into two parts or two currents: a right current which was continuous with the vessels of the fixed duodenum and a left current which prolonged the arterial circles of the stomach onto the duodenal bulb.

The SP is best seen if grasping forceps are placed on the middle of the first part of the duodenum and then retracted downwards and towards the left. If the first part of the duodenum is short and twists to 

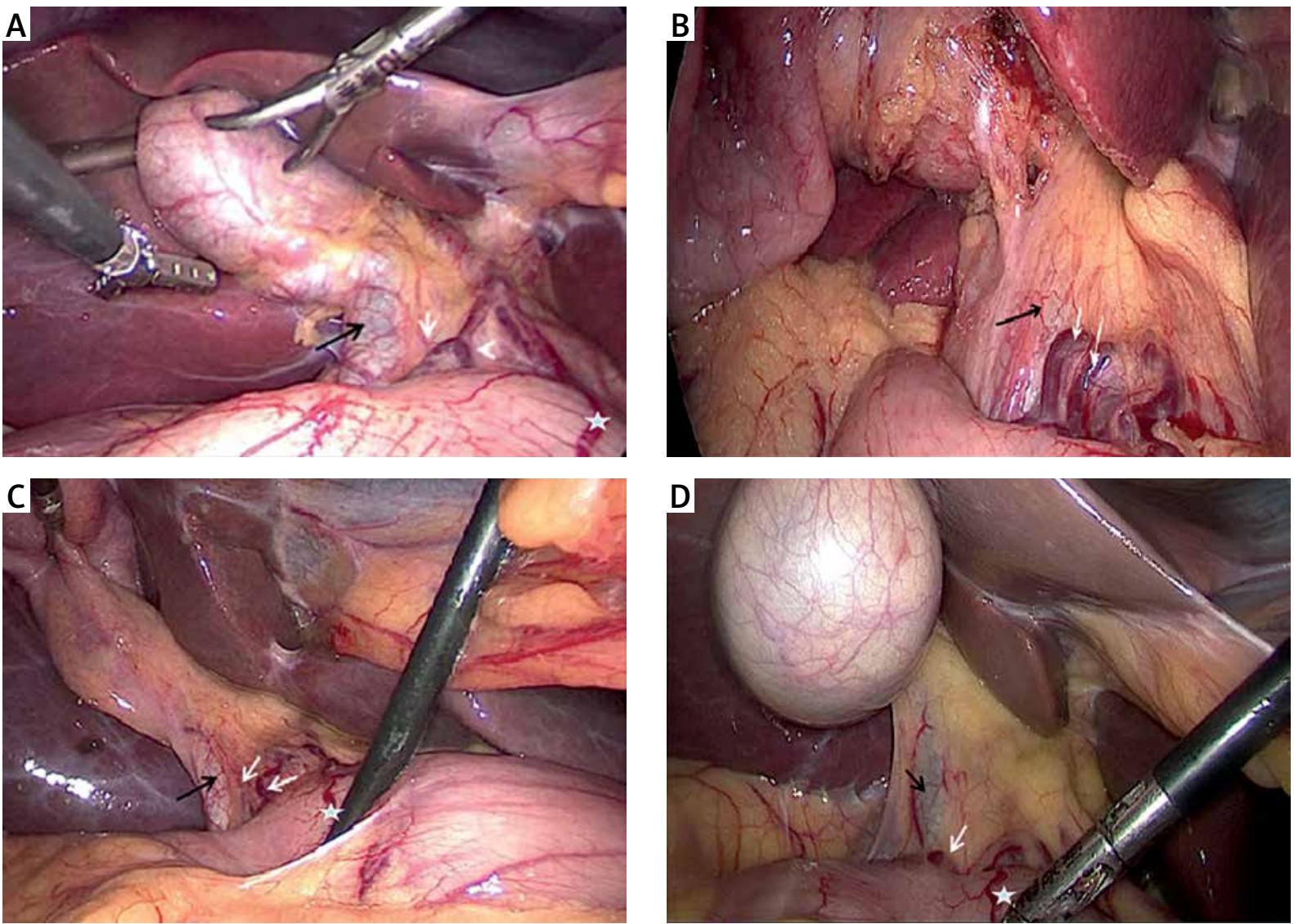

Photo 1. The white arrows show different type of supraduodenal pedicle. The CBD (black arrows) locate at the right and dorsal region of the rightmost branch of supraduodenal pedicle. Five-pointed star shows prepyloric vein

the right and dorsally, it is important to identify the prepyloric vein to avoid misidentifying the pylorus as the first part of the duodenum.

The supraduodenal pedicle was recognizable in 100/122 (82\%) consecutive patients undergoing LC in this hospital. These patients were operated on or supervised by one surgeon (LW). The SP displayed either thin $(9 / 100,9 \%)$ or obvious thick vessels (91/100, 91\%), 38/100 cases (38\%) showed a single branch vessel and $62 / 100$ cases (62\%) showed two or more ranches. The CBD and SP were both present in $68 / 100(68 \%)$ patients. The right most branch of the SP ran along the ventral $(40 / 68,58.8 \%)$ or the left edge $(28 / 68,41.2 \%)$ of the CBD. In 32 cases with an invisible CBD but a visible SP, the CBD was successfully found close to the right most branch of the SP in all patients after slightly separating them (Photo 1 ).

The SP can be a useful landmark to quickly and accurately locate the CBD in LC. The CBD is almost always located at the right or dorsal region of the rightmost branch of these vessels (belonging to the right current of the pedicle). It can also apply to laparoscopic common bile duct exploration (LCBDE).

\section{Conflict of interest}

The authors declare no conflict of interest.

\section{References}

1. Rystedt J, Lindell G, Montgomery A. Bile duct injuries associated with 55,134 cholecystectomies: treatment and outcome from a national perspective. World J Surg 2016; 40: 73-80.

2. Barrett M, Asbun HJ, Chien HL, et al. Bile duct injury and morbidity following cholecystectomy: a need for improvement. Surg Endosc 2018; 32: 1683-8.

3. Wang L, Zhou D, Hou H, et al. Application of "three lines and one plane" as anatomic landmarks in laparoscopic surgery for bile duct stones. Medicine 2018; 97: e0155.

4. Hentati N, Fournier HD, Papon X. Arterial supply of the duodenal bulb: an anatomoclinical study. Surg Radiol Anat 1999; 21: 159-64.

Received: 9.06.2021, accepted: 28.06.2021. 